\title{
Contribución al debate metodológico en salud
}

\author{
Contributions to the methodological debate on health
}

Estas páginas deben leerse como un intento de abrir algunas líneas de reflexión sobre la metodología de la historia, cuestión a mi criterio relativamente desdeñada por los historiadores argentinos. El problema se hace particularmente evidente cuando se observa la reciente expansión de una línea de trabajo que es identificada por diferentes investigadores en términos de Historia social de la salud y la enfermedad. Muy prolífica en los últimos años, ha tejido vínculos con múltiples investigadores en el país y en el exterior, organiza encuentros y jornadas en diferentes puntos del país con una recepción muy positiva si se tiene en cuenta la cantidad significativa de ponencias y asistentes que convoca, y se ha expresado a través de un buen número de publicaciones.

Si bien es justo reconocer esos logros, también merece alguna advertencia la creciente complejidad del campo, que superpone corrientes analíticas anteriormente diferenciadas como la historia de la medicina, la historia social de los procesos de salud/enfermedad/atención médica, la historia de las políticas de salud, la historia de los servicios de atención médica, la historia de las profesiones de salud y algunas otras que seguramente olvido.

Mi advertencia propone considerar que la suma de varias debilidades no siempre construye fortaleza. Bajo coordenadas tan heterogéneas el debate metodológico no solo se complejiza, se hace inabordable. $Y$ en tanto el abordaje metodológico se diferencia -legítimamente- según el objeto de estudio específico, las dificultades para el referato de artículos, la aceptación de ponencias e incluso la evaluación de tesis de maestría y doctorado, se vuelven insuperables. Todavía más grave: se dificulta considerablemente el debate entre investigadores.

Las dificultades que presenta la convivencia de objetos de investigación heterogéneos en el interior de un mismo campo de trabajo se agudiza cuando la metodología no se explicita. Me parece evidente -si alguien quiere desmentirme su palabra será bienvenida- que si se hace referencia a una historia social, es legítimo esperar la incorporación de alguna perspectiva de teoría social en la delimitación del objeto de estudio. No niego que esa incorporación se realice en un porcentaje importante de casos, pero en términos generales me atrevería a sostener que no se hace explícita, ni en la enumeración de los objetivos de conocimiento, ni en la construcción del objeto, ni en la formulación de las hipótesis que explicarían la dinámica de los procesos bajo análisis.

Soy plenamente consciente que sería abusivo afirmar que los historiadores contemporáneos desdeñan la teoría social. Se trata de una cuestión de matices, que sin embargo tiene una importancia clave en la construcción del texto. Los historiadores en general utilizamos conceptos teóricos o categorías para ordenar nuestro relato, para apuntalar conclusiones, para reforzar argumentos, para hacer más efectiva la comunicación de una reflexión compleja. También hablando en términos generales, a mi criterio no los utilizamos en medida suficiente para definir desde el comienzo mismo de la investigación las coordenadas que darán forma a nuestros objetos de estudio.

La perspectiva que quiero plantear en esta oportunidad remite a un posicionamiento relativamente frecuente en las ciencias sociales, que ha conducido a algunos analistas o metodólogos a postular la existencia de lo que caracterizan como un giro histórico. 
En palabras de un reconocido exponente de esa orientación metodológica, Terence McDonald:

\begin{abstract}
...uno de los aspectos diferenciadores de las actuales corrientes intelectuales es un giro hacia la "historia", que avanza en las humanidades y ciencias sociales contemporáneas. [Para este analista] ...los signos de una transformación significativa de las agendas intelectuales en las ciencias humanas serían evidentes en la aparición, ente otras innovaciones, del nuevo historicismo en literatura y teoría legal, un interés recuperado por la historia en filosofía, un nuevo institucionalismo históricamente orientado en ciencia política, una vertiente etnohistórica en antropología, de la sociología histórica en el respectivo campo e incluso una discusión metodológica más reflexiva e historicista en el interior de la misma historia. (1 p.5)
\end{abstract}

Esta transformación sería en sí misma histórica en tres sentidos:

1. Representa un cambio de época en relación a una ciencia social constituida al menos en parte en oposición a la historia, en los años de la inmediata posguerra.

2. Contiene un polémico y escasamente definido giro hacia la historia, no solo ni fundamentalmente como disciplina, sino como pasado, proceso y contexto, y su inclusión como un componente de investigaciones sobre una variedad de campos.

3. Está generando un interrogante renovado en la construcción de discursos e investigaciones disciplinarios en el campo de la historia.

La principal justificación de este esfuerzo de diferentes disciplinas por buscar en el pasado las lógicas constitutivas de los problemas contemporáneos puede expresarse en pocas palabras. En muchas de estas áreas se apuesta a una mayor capacidad explicativa que sería propia del relato histórico. En esos términos ese relato puede estar dotado de una capacidad hermenéutica que no siempre los mismos historiadores reconocen o utilizan en todo su potencial.

Hay una frase muy simple que resume este tipo de esfuerzos basados en el potencial hermenéutico de la reconstrucción histórica: la historia importa porque la historia explica. En esa lectura, la sociedad es abordada como una construcción, producto de la interacción entre sus miembros. Los procesos históricos son constitutivos; en su desenvolvimiento pueden por lo tanto encontrarse explicaciones y delimitarse influencias sobre las relaciones e identidades sociales resultantes.

La cuestión es abordada por diferentes analistas, cada uno incorporando su propio matiz. Paul Pierson por ejemplo señala la forma en que:

\footnotetext{
Muchos cientistas sociales asumen una perspectiva fotográfica de la vida política. Sus interrogantes giran en torno a una relación entre variables que interactúan en el presente: ¿cómo afecta los resultados políticos la distribución de la opinión pública?; ¿cómo las características sociales del individuo influencian la propensión a votar?; ¿cómo las reglas electorales afectan la estructura de los sistemas de partido? Las disputas entre teorías en competencia se centran en torno a qué factores en el entorno actual generan resultados políticos de consideración. Los análisis centrados en variables se basan, sin embargo, en su opinión, sobre algunos supuestos cuestionables en torno a la forma como opera el mundo social. El significado de tales "variables" resultaría frecuentemente distorsionado cuando son sacadas de su contexto temporal. Se sostiene por consiguiente un argumento fuerte para moverse desde la fotografía a los cuadros móviles. Ubicar la política en el tiempo -identificando sistemáticamente momentos particulares (incluyendo el presente) en una secuencia temporal de eventos y procesos- puede enriquecer considerablemente nuestra comprensión de una dinámica social compleja. (2 p.72)
}

El mismo Pierson, sin embargo, acota: "afirmar que la historia importa es insuficiente; los cientistas sociales quieren conocer porqué, dónde y cómo, se genera ese aporte específico". Y este es el desafío metodológico que debemos enfrentar si queremos validar el campo/los campos de investigación en salud. 
Demostrar que la historia explica es a mi criterio una tarea a la vez historiográfica y metodológica. Requiere de la descripción densa propia del relato histórico y de una construcción del objeto de investigación que se apoye decididamente sobre coordenadas teóricas.

Las secuencias que vinculan a determinadas cadenas de hechos en el tiempo no son evidentes para una mirada ingenua. El historiador debe sacarlas a la luz, y esta tarea se realiza de manera adecuada con utilización de teorías sociales afines a la construcción del problema de conocimiento que pretende iluminar.

En el campo específico de mi propia investigación -las políticas de salud y, específicamente, la historia de los servicios de atención médica- ese esfuerzo ha estimulado la aparición de estudios orientados a identificar procesos de mediana y larga duración en la construcción de instituciones de salud. En los abordajes más elaborados, la identificación se opera a través del análisis de las relaciones que unen a diferentes actores o agentes operando en un determinado sistema institucional, a los diferentes subsistemas entre sí, y a cada uno de ellos con otros actores en la sociedad civil y con las agencias del Estado. La reconstrucción histórica, con su minuciosa preocupación por los hechos en su encadenamiento temporal, constituye un insumo estratégico en este tipo de lecturas. Por esa vía el trabajo historiográfico sirve de apoyo, y a la vez se fortalece, con las nuevas preocupaciones y perspectivas teóricas emergentes en la sociología de las profesiones, en la economía institucional, en la ciencia política, en la teoría de las organizaciones.

\section{EL LUGAR DE LA TEORÍA EN MI TRABAJO HISTORIOGRÁFICO}

La búsqueda de una perspectiva teórica que potenciara la reconocida capacidad explicativa de la historia, me llevó en primer lugar a identificar actores con intereses en el sistema de seguridad social médica y a reconstruir sus enfrentamientos con una lógica de identidades e intereses en conflicto. Ese abordaje supone pensar las relaciones que se establecen en el interior del sector salud como fruto de la interacción entre agentes dotados de intereses y capacidades específicas y en condiciones de defender formas organizativas que suponen una determinada distribución de los recursos materiales y simbólicos disponibles en el mismo. En tanto el interlocutor principal para tales actores es el Estado, en función de su control de la capacidad -al menos teórica- de normatizar prácticas y organizaciones y reasignar recursos, la presencia de tales actores descubre, junto a referentes técnico-administrativos y financieros, una instancia que es fundamentalmente política.

Pese al reconocimiento de la importancia de la acción o inacción del Estado, el eje principal de ese análisis se colocó en la dinámica de los actores sociales y sus consecuencias sobre las instituciones que regulaban el funcionamiento del sistema de servicios, siguiendo, en parte mecánicamente, la orientación predominante en la bibliografía destinada a analizar diferentes aspectos del sistema político en Argentina.

El mismo límite presentó nuestra recuperación del marco legal-institucional en el que los actores desarrollaban su praxis. La articulación entre ese marco legal-institucional y las posibilidades de realización de los intereses de diferentes actores se elabora a nivel simbólico mediante la construcción de modelos, cada uno de ellos identificados por determinadas normas y su concreción en formas organizativas. Si los grupos y organizaciones insertos en el sector salud desarrollan cierta capacidad de acumulación de poder en función del control diferencial de determinadas reglas y recursos, la consolidación o ruptura de tales condiciones está fuertemente vinculada a la perduración o cambio de los modelos que regulan su distribución.

Cada modelo supone diferencias en la posición relativa de los actores y, particularmente, en el rol a jugar por el Estado, en el balance público/privado, en la redefinición de las organizaciones, de sus funciones y competencias. Los modelos no son más que abstracciones, formuladas sobre procesos sociales en permanente cambio, que orientan las estrategias de sus agentes. Cada iniciativa, cada demanda, cada decisión, cada nueva modalidad organizativa, pueden ser pensadas en función del reforzamiento o la negación de algunos de los modelos posibles. La referencia a modelos defendidos por 
actores movilizados nos ubica en el plano que hemos definido como político: el enfrentamiento de fuerzas sociales que procuran una determinada organización para un campo de objetos portadores de sentido. La identificación con determinados modelos es utilizada frecuentemente como recurso ideológico de legitimación de las demandas de los diferentes actores.

Cuando despuntaban los años '90, mi continuado interés en la evolución del sistema de servicios se vio potenciado por una circunstancia muy particular: el reconocimiento del proceso de emergencia de un nuevo régimen de regulación destinado a su reordenamiento. Cuando mi problema de investigación se focalizó en analizar las causas y condiciones del agotamiento de las formas de organización y financiamiento de la atención médica afianzadas a partir de los años '40 y los procesos que condujeron a su transformación, se hicieron evidentes los límites de la perspectiva teórica que acabo de sintetizar. Mi preocupación por los actores descuidaba la necesaria mirada de lo que ocurría en el interior del aparato estatal. El análisis de las demandas elevadas desde la sociedad civil hacia el Estado y los resultados de la acción o inacción de su aparato sobre la organización del sistema de servicios no prestaba adecuada atención a los procesos de toma de decisiones generados en el interior de la agencia estatal involucrada.

Ese abordaje del Estado como una caja negra cuyo contenido no se devela, y el estudio de los procesos de toma de decisiones en su interior, Ilevado adelante en función de considerar exclusivamente los inputs colocados por los actores en la sociedad civil y los outputs concretados en las decisiones emanadas de las respectivas agencias, se ubica en una orientación analítica que algunos cientistas políticos caracterizan como una perspectiva centrada en la sociedad. En la evaluación de Jonathan Fox (3), por ejemplo, los enfoques sociocéntricos explican la acción del Estado sobre la base de los intereses, relaciones y estructuras de la sociedad civil. Algunos enfatizan la influencia que las fuerzas sociales ejercen directamente sobre el Estado, mientras otros iluminan las restricciones externas que ellas imponen. Entre las limitaciones propias de ese enfoque se señala el reduccionismo resultante de atribuir exclusivamente a la intervención externa toda acción significativa por parte de sus agencias. No se considera que los actores estatales tienen sus propios intereses económicos y políticos, como individuos y como grupo. También tienen sus propias perspectivas sobre cómo responder a los desafíos desde dentro y fuera del Estado, y su control sobre las organizaciones estatales a menudo les proporciona la capacidad para poner en práctica esas ideas. Las perspectivas sociocéntricas fallarían por consiguiente en tanto no reconocen que los actores estatales pueden tener la disposición y capacidad para iniciar y proseguir sus propios intereses entre fuerzas sociales contrapuestas.

La propuestas superadoras tanto de perspectivas sociocéntricas como de aquellas que colocan el énfasis en la forma en que los líderes y la burocracia estatales defienden sus propios intereses o sus propias visiones y a partir de ellos modelan el orden social, proponen como alternativa de análisis la construcción de un enfoque relacional. Esto supone avanzar más allá de las simples lecturas instrumentalistas o subjetivistas del Estado, adoptando una perspectiva relacional y coyuntural que lo analice a partir de la forma que adquiere el conflicto entre fuerzas sociales en competencia por sus decisiones (4).

Un enfoque relacional requiere dar cuenta de cómo las capacidades de diferentes actores para alcanzar sus objetivos cambian a través del conflicto y la negociación. Tanto las respuestas que el Estado pone en marcha frente a la movilización de los actores sociales como las formas en que estos procesos se desenvuelven no estarían, conforme a esa perspectiva, predeterminadas por la distribución inicial de recursos de poder. Por el contrario, la fuerza o debilidad de las fuerzas pro-reforma es modelada a través de su interacción estratégica unas con otras y con sus oponentes.

El interés por este tipo de perspectiva no se agota sin embargo en una receta simple de incorporar actores estatales al anterior análisis de actores sociales, por el contrario, abre nuevos problemas teóricos y empíricos y el ensayo de nuevas categorías para dar cuenta de la continuidad y el cambio históricos. Muy particularmente los trabajos del neoinstitucionalismo histórico enfatizan algunos enfoques que pueden orientar el análisis de las políticas de salud en Argentina: ejemplos claves serían la introducción de esquemas configurativos que intentan recuperar la multiplicidad de factores que inciden en el diseño de nuevas instituciones, el abordaje de coyunturas críticas, la emergencia de legados históricos o procesos path dependent. 
Katznelson, por ejemplo, recupera los aportes del institucionalismo histórico a partir de la manera en que conecta el diseño institucional a la formación y existencia de agentes políticos portadores de preferencias, intereses e identidades particulares (5). En ese sentido recupera el texto de Theda Skocpol, Protecting soldiers and mothers (6), en tanto procura comprender la manera en la cual las instituciones políticas y las políticas modelan intercambios de doble vía entre Estado y sociedad civil. Por ese camino Skocpol enriquecería el análisis macro, habitual en los años '60 y '70, a partir de incorporarle los matices y el detalle propios de los trabajos históricos sobre economía política y política pública.

Un concepto clave es el de trama institucional. Su incorporación supone pensar la lucha política más allá de una contraposición de fuerzas en espacios de confrontación y negociación que se redefinen continuamente en función de los intereses y estrategias de los principales actores, tal como se expresa en la imagen de un mercado político. Se insertaría por el contrario en el interior de una trama institucional con capacidad para establecer las reglas de juego para la confrontación y permitir la elaboración de acuerdos relativamente estables. Las capacidades institucionales del Estado serían cruciales en la construcción y reproducción de esa trama. Su falta de coherencia, su inestabilidad, la inexistencia de capacidad de imponer sanciones o el no reconocimiento de su legitimidad por parte de actores con capacidad de veto, implicarían déficit significativos en la capacidad de gobierno por parte de las instituciones estatales.

Diferentes autores señalan la conveniencia de focalizar el análisis en el interior de cada una de las arenas de política. En torno a cada política pública se organizaría una trama institucional que nuclea actores sociales y estatales con recursos, intereses e ideas diferentes. Todos los actores relevantes interactúan con racionalidad limitada y en condiciones de incertidumbre, por lo que se requieren mecanismos colectivamente aceptados que den forma a sus intercambios. La capacidad de gestión, en ese marco, refiere a la posibilidad de poner en marcha tales mecanismos ordenadores de las:

...diversas y múltiples interacciones que llevan adelante, a través de períodos prolongados y en ámbitos específicos de políticas públicas, los actores relevantes de la sociedad y el Estado, los cuales actúan estratégicamente guiados por intereses y cosmovisiones ideológicas potencialmente conflictivas entre sí. (7)

El análisis institucional, por consiguiente, en tanto perspectiva relacional entre Estado y sociedad, permitiría no solo entender la interacción de actores estratégicos con intereses e ideas muchas veces contradictorias, sino calibrar y ponderar las formas en que se retroalimentan la gobernabilidad y la gestión pública o capacidad de gobierno. Esa articulación no es dada, por el contrario, se trata de una construcción social, no solo generada por el juego político, sino también conservada o modificada; puede por consiguiente variar en las diferentes áreas de acción pública e incluso en un área determinada a través del tiempo.

Los intercambios entre actores involucrados en diferentes políticas públicas dependen de la calidad de los respectivos contextos institucionales. En consecuencia serían las instituciones en tanto reglas, normas, prácticas informales y tradiciones que resultan de la acción humana, las que estructuran y dan cuerpo a las relaciones entre Estado y sociedad. Tales relaciones están sujetas a diferentes ritmos de cambio: en tiempos normales de la vida política la transformación se produce de manera incremental y en el margen; en épocas de crisis las mutaciones se aceleran en razón de los excesivos costos que representa para determinados actores mantener los viejos mecanismos de interacción.

La identificación de diferentes ritmos y diferente profundidad en el cambio institucional estimuló la recuperación de coyunturas críticas. El cambio incremental se asocia a un proceso relativamente continuo a partir de la agregación de decisiones tomadas por actores individuales a lo largo del tiempo. El cambio que genera transformación en las reglas de juego suele ser más discontinuo, es introducido a través de decisiones tomadas a nivel macro y conduce a la fundación de nuevos órdenes legales, agencias estatales $u$ otros arreglos institucionales. Los analistas del cambio social suelen centrar su interés en los períodos en que se generan estas transformaciones argumentando que establecen ciertas direcciones de cambio y cierran otras en una forma que modela la política de los años futuros. Tales transiciones reciben la denominación de coyunturas críticas (8). 
El carácter de las coyunturas críticas y la perspectiva desde la cual son analizadas varía considerablemente. Sin embargo, subyaciendo a esta diversidad es posible reconocer una comprensión común del cambio que ha constituido un hito de la investigación histórica comparativa sobre el desarrollo. Sugiere lo que se ha llamado un patrón de cambio path dependent, en el que los resultados que se generan durante una transición de este nivel de profundidad promueven diferentes trayectorias en las políticas futuras.

De ese modo el análisis de las coyunturas críticas permite identificar la génesis y los mecanismos de afirmación de legados históricos o secuencias path dependent. Ambos conceptos remiten a una determinada forma de relación entre actores sociales y actores estatales que a posteriori puede ser reconocida como un rasgo persistente en la arena política involucrada. Las instituciones forjadas en una determinada coyuntura pueden definir tales senderos, estructurando la posterior respuesta ante nuevos desafíos. En sus primeros análisis los institucionalistas históricos enfatizaron el impacto de las capacidades estatales y los legados de política sobre las subsiguientes opciones de elaboración e implementación de políticas. Otros enfatizan el modo en que las políticas sancionadas condicionan el futuro conflicto político estimulando a las fuerzas sociales a organizarse, enfrentarse o negociar bajo determinadas formas, a redefinir sus identidades o a desarrollar determinados intereses en áreas políticas que resultará problemático modificar. En este contexto, se reconoce la presencia de consecuencias no esperadas e ineficiencias generadas por las instituciones existentes en contraste con otras imágenes que dan por cierta su capacidad de definir y afianzar reglas ordenadoras.

Pese a la importancia que otorgan a las instituciones, los institucionalistas históricos raramente las abordan como la única fuerza condicionante en una determinada política. En general han estado particularmente atentos a la relación entre instituciones e ideas o creencias (9). El análisis histórico comparado de cómo las relaciones entre Estados y ciudadanos y entre Estados y economías están contingentemente modeladas en el interior de conjuntos específicos de legados y diseños institucionales, ha vinculado efectivamente el trabajo empírico con cuestiones normativas básicas en la tradición liberal, sobre todo aquellas que conciernen a la representación de intereses. El foco en estos estudios ha girado hacia la manera en la cual las instituciones modelan la formación y agregación de intereses individuales y de grupo. Del mismo modo han rechazado la perspectiva que reduce la realidad a la significación, y a diferencia del enfoque de elección racional, con su reducción de la acción a individuos efectivamente utilitarios, identifican escenarios donde tanto el contenido como los medios de la acción varían según configuraciones organizativas, culturales e históricas.

En algunos textos se evidencia una preocupación por aplicar las ideas vinculadas a path dependency al análisis de procesos sociales de una manera que explicite más concretamente los mecanismos involucrados en la construcción y mantenimiento de un determinado legado. Esto significa focalizar en las dinámicas de los procesos auto-reforzadores en el interior de un determinado sistema político. La hipótesis de trabajo es que el movimiento inicial en una dirección particular estimula posteriores movimientos a lo largo del mismo camino. A lo largo del tiempo el camino no elegido se convierte en una alternativa crecientemente distante, crecientemente inabordable. Considerados ex ante, tales procesos pueden producir más de un resultado posible. Una vez que el camino particular se establece, sin embargo, los procesos autorreforzadores tienden a la consolidación o la institucionalización. Las coyunturas críticas generan caminos persistentes de construcción del conflicto político.

Se trata solo de una propuesta teórico-metodológica, sin duda no generalizable. Sin embargo, me pareció útil su explicitación en tanto considero que está en la base del interés por intentar construir una historia frente a la posibilidad de resignarse a recuperar una crónica. Ese intento no convierte a su ejecutor en depositario de la verdad histórica, por el contrario, permite generar debates en torno a las hipótesis, las categorías utilizadas, la eficacia del recorte propuesto.

Las crónicas solo alimentan un debate sobre los hechos, sean o no significativos. Si se define un entramado de relaciones, solo serán pertinentes los hechos que puedan incidir en esa trama. Esta afirmación, que puede parecer obvia, se hace necesaria cuando se observa la utilización de determinadas fuentes por algunos historiadores. Por eso es muy importante que el historiador demuestre en su análisis el interés específico de la fuente que utiliza para el proceso histórico que busca reconstruir. 
Con esto recupero mi preocupación sobre la complejidad de la línea de investigación que se propone con la referencia a una Historia social de la salud y la enfermedad. Para utilizar un ejemplo extremo que me permita explicitar mi preocupación en forma breve: si un historiador está preocupado por la trayectoria académica de un científico, solo agregará nuevo conocimiento al campo de estudio de la salud pública o la salud colectiva si puede demostrar que los tropiezos o errores en ese campo tuvieron repercusión a nivel macro, ya sea en el campo de las políticas o de la organización del sistema de servicios.

Susana Belmartino

Doctora en Historia, Université d'Aix-Marsella, Francia. Docente-Investigadora, Universidad Nacional de Rosario (UNR), Argentina.

\section{REFERENCIAS BIBLIOGRÁFICAS}

1. McDonald TJ, editor. The Historic Turn in the Human Sciences. Michigan: University Press; 1999.

2. Pierson P. Not just what, but when: timing and sequence in political processes. Studies in American Political Development. 2000;14(1):72-92.

3. Fox J. State-Society interaction and distributive reform in Mexico. En: The Politics of Food in Mexico. State Power and Social Mobilization. Ithaca, Londres: Cornell University Press; 1993.

4. Jessop B. Towards a theoretical account of the State. En: The Capitalist State. Marxist Theories and Methods. Nueva York, Londres: New York University Press; 1982.

5. Katznelson I. Structure and configuration in comparative politics. En: Lichbach MI, Zuckerman AS, editores. Comparative politics. rationality, culture and structure. Cambridge: Cambridge University Press; 1997. p. 81-112.
6. Skocpol Th. Protecting soldiers and mothers. Cambridge: Harvard University Press; 1992.

7. Repetto F. Gobernabilidad y capacidad de gestión pública: las políticas frente a la pobreza en Chile y Argentina (1990-1996). En: Labastida J, editor. Gobernabilidad en América Latina. México DF: Instituto de Investigaciones Sociales (UNAM), FLACSO México, Plaza y Valdés; 2001.

8. Collier RB, Collier D. Shaping the political arena. Critical junctures, the labor movement and regime dynamics in Latin America. Princeton: Princeton University Press; 1991.

9. Hall P, Taylor R. Political Science and the three new Institutionalisms. [En línea] Max Planck Institut für Gesellschaftsforschung. Alemania: MPIFG; 1996. (Discussion Paper 96/6) [fecha de acceso 10 de junio de 2008] URL disponible en: http://www.mpifg.de/pu/mpifg_dp/dp96-6.pdf

\section{FORMA DE CITAR}

Belmartino S. Contribuciones al debate metodológico en salud. [Editorial]. Salud Colectiva. 2008;4(2):125-131. 